\title{
Odontogenic Myxoma Located in The Mandible: A Case Report
}

\author{
Ferhat Ayranc1 ${ }^{1}$, Mehmet Melih Ömezli ${ }^{1}$, Orhan Zeki Rastgeldi ${ }^{1}$, Aslıhan Duman ${ }^{2}$ \\ ${ }^{1}$ Department of Oral and Maxillofacial Surgery, Faculty of Dentistry, Ordu University, 52100, Ordu, Turkey \\ ${ }^{2}$ Department of Pathology, Faculty of Medicine, Giresun University; 28500, Giresun, Turkey,
}

Received: 11 November 2015 accepted: 12 November 2015/ published online: 24 December 2015

(C) Ordu University Institute of Health Science, Turkey, 2015

\begin{abstract}
Odontogenic myxomas are benign but locally invasive tumor originating from primordial mesenchymal tooth forming tissues which do not metastasise. They can be found in both the maxilla and mandible, usually associated with a tooth germ. It occurs mostly in the second or third decades of life and affects mainly the posterior mandible. Radiographically it appears as a unilocular or multilocular radiolucency with irregular margins. They are usually asymptomatic, with the potential to attain great size without noticeable signs and symptoms. Nevertheless, in the current literature, several symptoms have been associated with odontogenic myxomas: tooth displacement and mobility, malocclusion, facial asymmetry, delayed eruption of teeth, disturbance of speech and mastication,pain, and mandibular nerve paresthesia. They are rare tumors and account for 3.3-15.7\% of all odontogenic tumors in adults.

In this case report, a case of Odontogenic myxoma $(\mathrm{OM})$ localized in the mandible was presented.

Key words: Enucleation, Mandible, Odontogenic myxoma.

Address for correspondence/reprints:

Orhan Zeki Rastgeldi

E-mail address: e-mail.zekirastgeldi@hotmail.com

*In this case report, a case of Odontogenic myxoma localized in the mandible was presented.

DOI: $10.19127 /$ mbsjohs.08256

\section{Introduction}

Odontogenic myxoma (OM) is a benign, locally invasive and aggressive, non-metastasizing neoplasm of the jaw bones (Leiser et al., 2009). It is a slow growing tumor consisting of an accumulation of mucoid ground substance with little collagen, the amount of which determines whether it can be called a myxofibroma (Simon et al., 2004). Histopathologically these benign neoplasms were classified by the World Health Organization as benign odontogenic neoplasms of ecto-mesenchymal origin consisting of rounded and angular cells embedded in an abundant myxoid stroma with few collagen fibrils probably originating from either the dental papilla follicle or periodontal ligament (Mayrink et al., 2013).
\end{abstract}


OMs are one of the most common lesions of jaw bones, ranging from about 3\% to $8 \%$ of odontogenic tumors and cysts (Keszler et al., 1995). Most of the OMs reported were young adults affected mostly in their second and third decade of life with marked female predilection (Manne et al., 2012). OM is generally depicted as slow growing tumor with the potential to attain considerable size without noticeable signs and symptoms. The molar and ramus regions of the mandible are most frequently involved, whereas the premolar-first molar region is the site of predilection in the maxilla (Noffke et al., 2007). The possible surgical management can vary from simple curettage and peripheral ostectomy up to segmental resection (Leiser et al., 2009; Manne et al., 2012; Mayrink et al., 2013).

\section{Case}

A 33 year-old man presented to Ordu Faculty of Dentistry, Department of Oral and Maxillofacial Surgery for routine dental examination. Clinical examination revealed no facial asymmetry extra orally on inspection. On palpation, there was no swelling or tenderness present. The family history was non-contributory and there was no trauma history or any developmental abnormalities. In intraoral examination there was no buccal and lingual cortical plate expansion. Panoramic radiograph was obtained for radiographical examination. In the panoramic radiograph there was an uniocular radiolucencies at left mandible; from canine to mesial root of first molar (Fig 1).

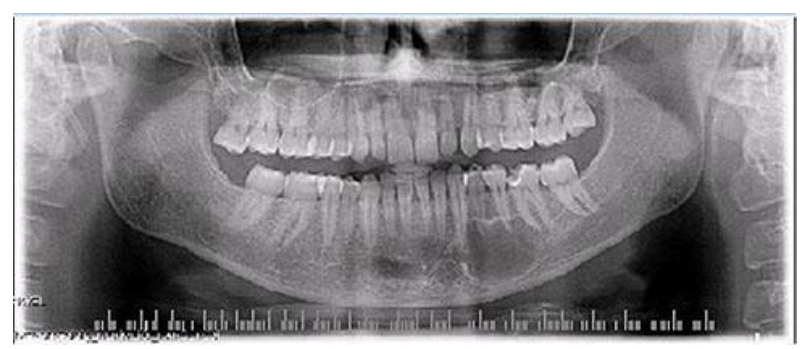

Figure 1: Preoperative OPG view showing uniocular radiolucent lesion involving mandibula.

Differential diagnosis of dentigerous cyst, odontogenic keratocysts, unicystic calcifying epithelial odontogenic tumor and ameloblastoma was considered. We decided to treat the lesion with associated non-vital teeth root resection and enucleation. After informing the patient of all possible complications that can occur during and after the surgery, a signed consent form was obtained from the patient. Under local anesthesia a mucoperiosteal flap was raised from the lower left mandible from canine to first molar. Bone was removed with a bur and adequate coolant.

The lesion was removed and premolars were resected from apical. The lesion was sent to pathological analysis (Fig 2-A). Pathological report showed that the lesion was odontogenic myxoma (Fig 2-B, C, D).
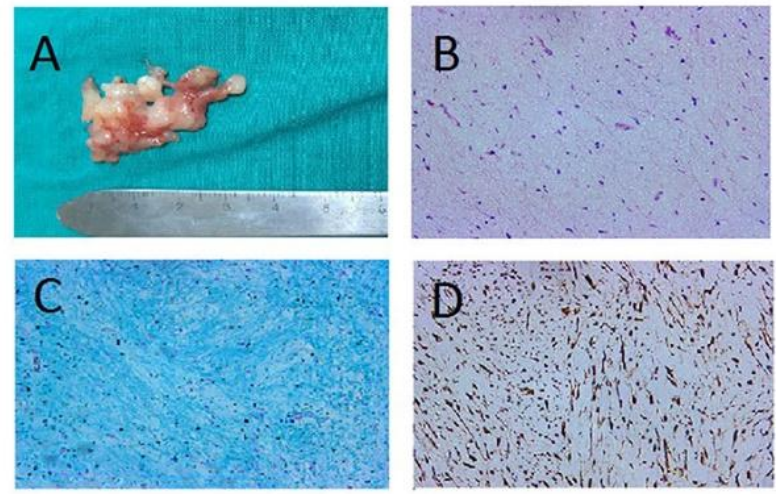

Figure 2: A) The lesion was removed. B) The histology is essentially identical to that seen in H\&E. C) Myxoid stroma stained with alcalin blue. D) The cells are immunoreactive with antibody against vimentin.

The surgical wound was closed primarily with silk sutures. Antibiotic therapy (amoxicillin+clavulanate, $1250 \mathrm{mg} /$ day), and an analgesic (paracetamol) were prescribed after surgery. The patient was reassessed 7 days after surgery. The sutures were removed with satisfactory wound healing. The postoperative course was uneventful and the patient was asymptomatic in the 15 months period of followup (Fig 3).

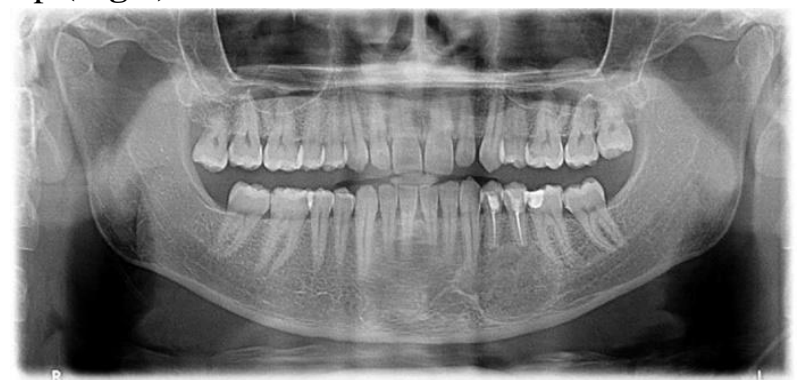

Figure 3: Postoperative OPG view 15 months after surgery.

\section{Discussion}

$\mathrm{OM}$ is regarded as a locally invasive tumor that does not metastasize and presents slow and asymptomatic expansion, sometimes resulting in perforation of the cortical borders of the affected 
bone (Zainine et al., 2013). In many cases, these lesions are diagnosed accidentally by a routine dental checkup appearing as a "soap bubble" (Kawase-Koga et al., 2014). Clinically, myxomas are benign, insidious lesions, often largely asymptomatic. Thus they most commonly appear as a painless swelling which can attain a large size before diagnosis (Halfpenny et al., 2000) Other symptoms tend to manifest later, as a consequence of expansion in relation to adjacent structures. These include pain or, especially in the maxilla, involvement of surrounding soft tissues (Halfpenny et al., 2000; Boffano et al., 2011; Zainine et al., 2013).

Radiographically, OMs frequently appear as unilocular or multilocular radiolucencies with well-defined margins and fine bony septa. The lesion sizes are correlated with their locularity (Kaffe et al., 1997; Morihiro Higo, 2015). Lesions $>40 \mathrm{~mm}$ tend to be multiloculated, and smaller lesions tend to be uniloculated (Kaffe et al., 1997; Morihiro Higo, 2015). The presentation often is describedas a honeycomb, soap-bubble, tennisracket, or ground-glass pattern (Morihiro Higo, 2015). Differential diagnosis like ameloblastoma, ameloblastic fibroma, odontogenic fibroma, central hemangioma, or odontogenic keratocyst along with odontogenic myxoma could be listed as initial diagnostic hypothesis based on the clinical and radiological findings (Manne et al., 2012). Computed tomography was more likely to display a cortex and its perforation, tooth displacement and root resorption were more reliably observed on conventional radiography (MacDonald-Jankowski et al., 2004).

$\mathrm{OM}$ is associated with a high rate of recurrence, due to its gelatinous nature and the absence of a capsule; prolonged clinical and radiological surveillance is therefore mandatory. The lesion is not radiosensitive and studies have shown no longterm cure with radiotherapy alone (Landa et al., 2002)

Radical surgery, excision, or enucleation followed by curettage of the surrounding bony tissue have all been advocated as treatment options ( Lo Muzio et al., 1996; Li et al., 2006; Rocha et al., 2009). Complete surgical removal by conservative treatment can be difficult, because, unlike most benign neoplasms, the myxoma is not encapsulated and its myxomatous tissue infiltrates the surrounding bony tissue without its immediate destruction (Lo Muzio et al., 1996). Conservative treatments like enucleation and curettage have several advantages over more radical treatments, such as segmental or block resection, and hemimandibulectomy with reconstruction surgery (Kawase-Koga et al., 2014). Conservative treatments are substantially less invasive, can be achieved by means of an intraoral surgical approach, preserve function and aesthetics, have a shorter hospitalization time, and are more costeffective (Rocha et al., 2009; Kawase-Koga et al., 2014). Nonetheless, the risk of recurrence after more conservative surgery is higher as the myxoma is not encapsulated and its myxomatous tissue infiltrates the surrounding bony tissue without causing immediate destruction (Colburn and Epker, 1975; Lo Muzio et al., 1996; Li et al., 2006; Rocha et al., 2009; Boffano et al., 2011; KawaseKoga et al., 2014). Few authors in the literature in the last 20 years with a consistent number of patients report on surgical treatment, follow-up, and recurrence of their study populations. According to Sloootweg's et al. (1986) 15 patients the maxilla was involved in 4 cases whereas the tumor was situated in the mandible in 11 cases. Of the 9 patients who underwent conservative treatment, one exhibited recurrent tumor. Six patients were treated by resection including uninvolved adjacent tissue; none of them has so far exhibited recurrence (Slootweg and Wittkampf, 1986). Li's identified that in their 5 cases treated conservatively by enucleation, the remaining 20 cases were treated by relatively radical procedures, including block/segmental resection and partial or total maxillectomy or mandibulectomy. Follow-up data were available on 22 patients and only 1 patient initially treated by enucleation had a recurrence (Li et al., 2006). According to Boffano et al. (2011) 3 patients of the 10 patient treated by enucleation and curettage. Instead, in the remaining 7 patients, segmental resection and immediate reconstruction were decided. At followup, no patient showed recurrence of the lesion (Boffano et al., 2011). In the mandible it should be possible to extirpate carefully all macroscopically visible turnout tissue by enucleation followed by curettage of the surrounding bone (Slootweg and Wittkampf, 1986). Perhaps a conservative approach may be used for smaller lesions to preserve function, reserving more radical surgery for recurrences and larger lesions (Lo Muzio et al., 1996; Kansy et al., 2012; Kawase-Koga et al., 2014).

\section{Conclusion}

Our protocol is to perform conservative surgery by enucleation and curettage when lesions were 
smaller than $3 \mathrm{~cm}$, whereas a segmental resection with immediate reconstruction is preferred in patients affected by bigger tumors. Long-term follow-up is required, in particular when conservative surgery is preferred.

Informed Consent: Written informed consent was obtained from patient who participated in this study.

Peer-review: Externally peer-reviewed.

Author Contributions: Concept, Design and Supervision FA; Data Collection and/or Processing MMO; Analysis and/or Interpretation AD; Literature Review OZR; Writing FA; Critical Review MMO.

Conflict of Interest: No conflict of interest was declared by the authors.

Financial Disclosure: The authors declared that this study hasn't received no financial support.

\section{References}

Boffano P, Gallesio C, Barreca A, Bianchi FA, Garzino-Demo P, Roccia F. Surgical treatment of odontogenic myxoma. The Journal of craniofacial surgery 2011;22: 982-7.

Colburn JF, Epker BN. Myxoma of the mandibular condyle--surgical excision with immediate reconstruction. J Oral Surg 1975; 33: 351-5.

Halfpenny W, Verey A, Bardsley V. Myxoma of the mandibular condyle. A case report and review of the literature. Oral Surg Oral Med Oral Pathol Oral Radiol Endod 2000; 90: 34853.

Kaffe I, Naor H, Buchner A. Clinical and radiological features of odontogenic myxoma of the jaws. Dento maxillo facial radiology 1997; 26: 299-303.

Kansy K, Juergens P, Krol Z, Paulussen M, Baumhoer D, Bruder E, Schneider J, Zeilhofer HF, Schwenzer-Zimmerer K. Odontogenic myxoma: diagnostic and therapeutic challenges in paediatric and adult patients--a case series and review of the literature. J Craniomaxillofac Surg 2012; 40: 271-6.

Kawase-Koga Y, Saijo H, Hoshi K, Takato T, Mori Y. Surgical management of odontogenic myxoma: a case report and review of the literature. BMC research notes 2014; 7: 214.

Keszler A, Dominguez FV, Giannunzio G. Myxoma in childhood: an analysis of 10 cases. J Oral Maxillofac Surg 1995; 53: 518-21.

Landa LE, Hedrick MH, Nepomuceno-Perez MC, Sotereanos GC. Recurrent myxoma of the zygoma: a case report. J Oral Maxillofac Surg 2002; 60: 704-8.
Leiser Y, Abu-El-Naaj I, Peled M. Odontogenic myxoma--a case series and review of the surgical management. J Craniomaxillofac Surg 2009; 37: 206-9.

Li TJ, Sun LS, Luo HY. Odontogenic myxoma: a clinicopathologic study of 25 cases. Archives of pathology \& laboratory medicine 2006; 130: 1799-806.

Lo Muzio L, Nocini P, Favia G, Procaccini M, Mignogna MD. Odontogenic myxoma of the jaws: a clinical, radiologic, immunohistochemical, and ultrastructural study. Oral Surg Oral Med Oral Pathol Oral Radiol Endod 1996; 82: 426-33.

MacDonald-Jankowski DS, Yeung RW, Li T, Lee KM. Computed tomography of odontogenic myxoma. Clin Radiol 2004; 59: 281-7.

Manne RK, Kumar VS, Venkata Sarath P, Anumula L, Mundlapudi S, Tanikonda R. Odontogenic myxoma of the mandible. Case Rep Dent 2012: 214704.

Mayrink G, Luna AH, Olate S, Asprino L, De Moraes M. Surgical treatment of odontogenic myxoma and facial deformity in the same procedure. Contemp Clin Dent 2013; 4: 390-2.

Morihiro Higo AK, Katsunori Ogawara, Masashi Shiiba, Katsuhiro Uzawa, Hideki Tanzawa. A case of a rapidly expanding odontogenic myxoma of the mandible. Oral Sci Int 2015; 12: 22-26.

Noffke CE, Raubenheimer EJ, Chabikuli NJ, Bouckaert MM. Odontogenic myxoma: review of the literature and report of 30 cases from South Africa. Oral Surg Oral Med Oral Pathol Oral Radiol Endod 2007; 104: 101-9.

Rocha AC, Gaujac C, Ceccheti MM, Amato-Filho G, Machado GG. Treatment of recurrent mandibular myxoma by curettage and cryotherapy after thirty years. Clinics 2009; 64: 149-52.

Simon EN, Merkx MA, Vuhahula E, Ngassapa D, Stoelinga PJ. Odontogenic myxoma: a clinicopathological study of 33 cases. Int J Oral Maxillofac Surg 2004; 33: 333-7.

Slootweg PJ, Wittkampf AR. Myxoma of the jaws. An analysis of 15 cases. Journal of maxillofacial surgery $1986 ; 14:$ 46-52.

Zainine R, Mizouni H, El Korbi A, Beltaief N, Sahtout S, Besbes G. Maxillary bone myxoma. Eur Ann Otorhinolaryngol Head Neck Dis 2013; 131: 257-9. 